\title{
HPV-Mediated (p16-Positive) Oropharyngeal Carcinoma by AJCC v8
}

\section{Stage}

National Cancer Institute

\section{Source}

National Cancer Institute. HPV-Mediated (p16-Positive) Oropharyngeal Carcinoma by A/CC v8 Stage. NCI Thesaurus. Code C132882.

A term that refers to the staging of HPV-mediated (p16-positive) oropharyngeal carcinoma according to the American Joint Committee on Cancer, 8th edition. 